\title{
Effects of Sea Spray on Tropical Cyclone Intensity
}

\author{
EDGAR L ANDREAS \\ U.S. Army Cold Regions Research and Engineering Laboratory, Hanover, New Hampshire
}

KERRY A. EMANUEL

Massachusetts Institute of Technology, Cambridge, Massachusetts

(Manuscript received 20 April 2000, in final form 14 May 2001)

\begin{abstract}
The intensity of tropical cyclones is sensitive to the rates at which enthalpy and momentum are transferred between sea and air in the high-wind core of the storm. Present models of the wind dependence of these transfer rates suggest that the effective drag coefficient is more than twice the effective enthalpy transfer coefficient at wind speeds above $25 \mathrm{~m} \mathrm{~s}^{-1}$. Using this ratio in numerical models, however, makes it impossible to sustain storms of greater than marginal hurricane intensity. Some other physical process must, therefore, enhance enthalpy transfer at very high wind speeds. This paper suggests that re-entrant sea spray explains this enhanced transfer. When a spray droplet is ejected from the ocean, it remains airborne long enough to cool to a temperature below the local air temperature but not long enough to evaporate an appreciable fraction of its mass. The spray droplet thus gives up sensible heat and returns to the sea before it has time to extract back from the atmosphere the heat necessary to continue its evaporation. Microphysical modeling, combined with data from the Humidity Exchange over the Sea Experiment (HEXOS), makes it possible to derive an expression for the net enthalpy transfer of re-entrant spray. This spray enthalpy flux is roughly cubic in wind speed. When this relation is used in a numerical simulation of a hurricane, the spray more than compensates for the observed increase in the ratio of drag and enthalpy transfer coefficients with wind speed. The momentum flux associated with sea spray is an important energy sink that moderates the effects of this spray enthalpy flux. Including a parameterization for this momentum sink along with wave drag and spray enthalpy transfer in the hurricane simulation produces results that are similar to ones based on equal transfer coefficients.
\end{abstract}

\section{Introduction}

Basic theory (e.g., Emanuel 1986) and numerical experiments (Ooyama 1969; Rosenthal 1971; Emanuel 1995) show that the intensity of tropical cyclones depends strongly on the coefficients for the transfers of momentum $\left(C_{D}\right)$ and enthalpy $\left(C_{k}\right)$ between the ocean and the atmospheric boundary layer. The maximum wind speed, in particular, depends on $\left(C_{k} / C_{D}\right)^{1 / 2}$ in the high-wind-speed core of the storm (Emanuel 1986). Unfortunately, there are no simultaneous measurements of the effective values of these coefficients at wind speeds greater than about $25 \mathrm{~m} \mathrm{~s}^{-1}$, and the theory of air-sea interaction at very high wind speeds is poorly developed. The agitated sea no doubt increases the effective roughness length and, thereby, $C_{D}$ and the dissipation rate of kinetic energy; while, for wind speeds up to about $20 \mathrm{~m} \mathrm{~s}^{-1}$, there is little observational evidence to suggest a corresponding increase in $C_{k}$ (Geernaert et al. 1987;

Corresponding author address: Dr. Edgar L Andreas, U.S. Army Cold Regions Research and Engineering Laboratory, 72 Lyme Road, Hanover, NH 03755-1290.

E-mail: eandreas@crrel.usace.army.mil
DeCosmo et al. 1996). Emanuel (1995) showed that, if estimated values of the exchange coefficients at $20 \mathrm{~m}$ $\mathrm{s}^{-1}$ applied at higher wind speeds, maintaining a storm of much greater than marginal hurricane intensity would be impossible. Some mechanism must also serve to enhance air-sea enthalpy exchange at high wind speed.

One candidate for enhancing the sea-air enthalpy flux at high wind speeds is sea spray. Riehl (1954, p. 287) was perhaps the first to suggest that sea spray supplies a significant amount of heat for generating and maintaining tropical storms. Laboratory studies (e.g., Mestayer and Lefauconnier 1988), numerical spray droplet models (e.g., Rouault et al. 1991; Edson et al. 1996; Van Eijk et al. 2001), and open-ocean observations (Korolev et al. 1990) all show that sea spray can redistribute enthalpy between the temperature and humidity fields in the marine boundary layer.

Fairall et al. (1994) were the first to incorporate a reasonable spray parameterization into a larger-scale cyclone model. While drawing no conclusions about whether spray had any effect on the intensity of their modeled storm, they did conclude that, without spray (or another mechanism for exchanging latent heat, such 
as rain), their model did not develop a realistic cyclone boundary layer. Kepert et al. (1999) continued this work and concluded that, although spray had little effect on the net air-sea enthalpy flux, it could increase storm intensity and affect development indirectly, by altering the stratification of the boundary layer, for instance. Lighthill et al. (1994) argued, however, that by reducing the surface layer temperature, evaporating sea spray would actually weaken tropical cyclones.

Because the net enthalpy flux across the air-sea interface, rather than the individual sensible and latent heat fluxes, controls a hurricane's intensity, Emanuel (1995) concluded that spray cannot affect the net enthalpy transfer. The marine boundary layer must supply all the latent heat required to evaporate the spray droplets. As we shall show here, however, Emanuel's (1995) conclusions hold only if all the sea spray evaporates. If some of the spray falls back into the sea-that is, is reentrant - an appreciable sea-air enthalpy flux results.

Three consecutive presentations at the American Meteorological Society's 23d Conference on Hurricanes and Tropical Meteorology further highlighted the diversity of opinions on spray's role in hurricane thermodynamics. In a preliminary version of this work, Andreas and Emanuel (1999) led off by showing that using an empirically based parameterization for the air-sea spray enthalpy flux in Emanuel's (1995) hurricane model produced a dramatic increase in storm intensity. Wang et al. (1999) followed with a more elaborate numerical model that incorporated spray through a parameterization based on Fairall et al. (1994). Wang et al. concluded that, although the presence of spray affected the structure of the boundary layer in their model and the rate at which their modeled cyclone intensified (cf. Kepert et al. 1999), it did not affect the storm's final intensity. Third in line was Uang (1999), who incorporated spray using the Fairall et al. parameterization in a hurricane model similar to Emanuel's (1995). Like Wang et al., Uang concluded that the spray had little effect on the final intensity of his modeled cyclones. Unlike Andreas and Emanuel (1999), however, these latter two presentations did not consider the effect of re-entrant spray.

At the 24th Hurricanes Conference, we reiterated our conclusion that spray is important for transferring enthalpy and momentum between the air and sea in high winds (Andreas and Emanuel 2000). Again, Wang et al. (2000) followed us immediately and showed that their modeled tropical cyclone evolved the same with or without spray when they used the Fairall et al. (1994) spray parameterization. They also showed, however, that when they used a spray parameterization based on Andreas and DeCosmo's (1999) spray model, their modeled cyclone became unrealistically intense. But they had misrepresented the Andreas and DeCosmo model such that their modeled air-sea enthalpy flux was too large. Naturally, their modeled storm became too intense. The irony in all these comparisons is that the Fairall et al. spray parameterization relies heavily on Andreas's
(1992) spray model, which is also the precursor of the Andreas and DeCosmo model and our current spray parameterization (Andreas and Emanuel 1999, 2000).

Recently, Bao et al. (2000) reported a numerical simulation of a developing hurricane in the Gulf of Mexico. They included the effects of spray in their simulation using the Fairall et al. (1994) algorithm but also tested our ideas about re-entrant spray (Andreas and Emanuel 1999). They found that, if all the spray evaporates in the marine boundary layer, the spray has a negligible effect on storm intensity, as Emanuel (1995) suggested. But if some of the spray falls back into the sea, there is a marked and realistic increase in the storm intensity, as we contend (Andreas and Emanuel 1999, 2000).

Here we continue our study of the air-sea enthalpy flux when the sea surface is producing copious spray. In section 2 , we show theoretically how spray can produce a net enthalpy flux from the ocean to the atmosphere. A small part of this net flux arises from the fact that the sea is generally warmer than the overlying air, so spray transfers some sensible heat directly to the atmosphere. A much larger enthalpy flux occurs when some of the spray falls back into the sea; these droplets have cooled below the air temperature but return to the sea before they have had time to extract from the atmosphere the sensible heat necessary to evaporate. This re-entrant spray thus cools the ocean and represents a net enthalpy flux to the atmosphere.

Using the spray model that Andreas (1992) developed and that Andreas and DeCosmo (1999) tuned with eddycorrelation measurements of the sensible and latent heat fluxes during the Humidity Exchange over the Sea experiment (HEXOS), we estimate the magnitude of spray's role in air-sea heat exchange in section 3. From the HEXOS observations, which include flux measurements in $10-\mathrm{m}$ winds up to $18 \mathrm{~m} \mathrm{~s}^{-1}$, we have shown that the spray contribution can, at times, exceed $100 \mathrm{~W}$ $\mathrm{m}^{-2}$ out of a combined surface sensible and latent heat flux (i.e., the net enthalpy flux) of $400 \mathrm{~W} \mathrm{~m}^{-2}$ (Andreas and Emanuel 1999). At much higher wind speeds, the spray contribution to enthalpy exchange will be an even larger fraction of the total because the spray enthalpy flux increases as the cube of the friction velocity.

In addition to providing an enhanced enthalpy flux, sea spray also extracts momentum from the wind (section 4). As spray droplets are ripped off the wave crest and injected into the airstream, they accelerate horizontally and, thus, represent a drag on the airflow. To our knowledge, we are the first to include spray momentum in a tropical cyclone simulation.

Finally, in section 5, we put all these ideas together in a series of tropical cyclone simulations using Emanuel's (1995) simple hurricane model. Incorporating realistic wave drag with a Charnock parameterization for surface roughness slows the wind and does not let the storm reach its full potential. Adding the spray enthalpy flux, however, provides the energy for a very intense hurricane. The spray momentum exchange can also be 


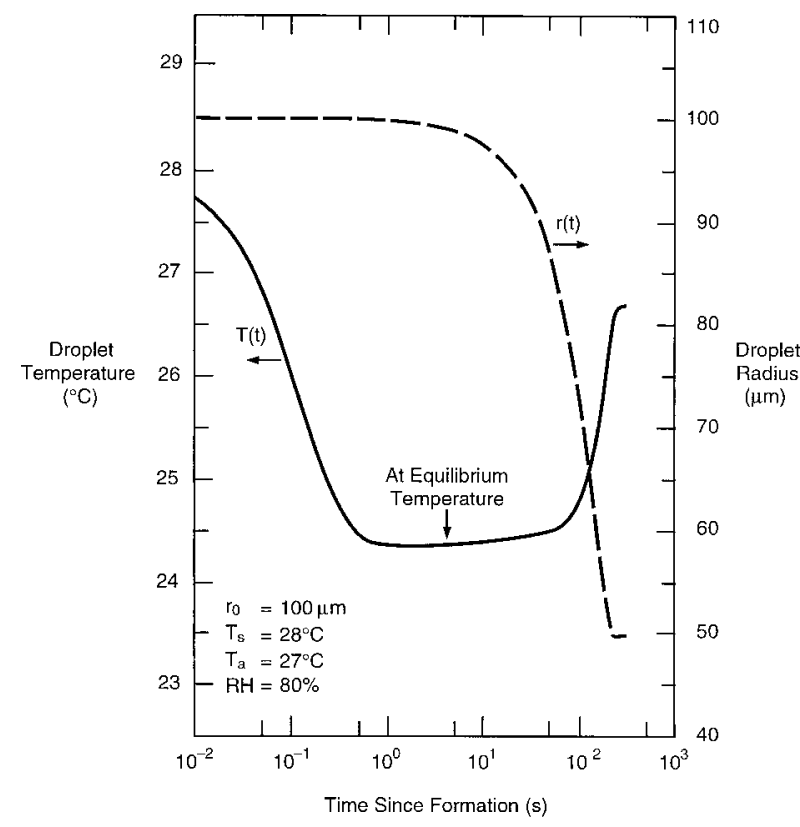

FIG. 1. The temperature $(T)$ and radius $(r)$ evolution of a spray droplet of initial radius $100 \mu \mathrm{m}$ that is ejected from the sea surface (at temperature $T_{s}=28^{\circ} \mathrm{C}$ ) into air of $27^{\circ} \mathrm{C}$ and $80 \%$ relative humidity. Also, the droplet has initial salinity 34 psu, and the barometric pressure is $1000 \mathrm{mb}$.

substantial and offsets some of the effects of the enhanced spray enthalpy flux. Combining the spray and wave-drag parameterizations produces a simulation with a cyclone intensity similar to our "control" run, which uses Emanuel's (1995) standard $C_{D}$ and $C_{k}$ values that are equal and increase linearly with the wind. Hence, spray-mediated exchange is one possible explanation why this simple parameterization produces realistic hurricanes (e.g., see Emanuel 1999).

\section{The thermodynamics of spray}

Microphysical modeling (e.g., Andreas 1990, 1992) has provided many insights into the processes that control air-sea heat and moisture transfer over a wind-agitated sea. The sea spray droplets that form from whitecaps and spume (e.g., Monahan et al. 1986) in such conditions, and that are important in transferring heat and moisture, range in radius from 1 to $500 \mu \mathrm{m}$. For this entire size range, however, the transfers of sensible and latent heat that these droplets facilitate are decoupled; that is, the sensible heat exchange that they mediate occurs three orders of magnitude more rapidly than the latent heat exchange.

Figure 1 demonstrates this for a $100-\mu$ m-radius spray droplet ejected into typical tropical cyclone conditions. In the figure, the droplet falls from $28^{\circ} \mathrm{C}$, the sea surface temperature, to its so-called equilibrium temperature within $1 \mathrm{~s}$. Only about $1 \%$ of the mass must evaporate for the droplet to reach this equilibrium temperature, as we will demonstrate shortly. Notice, too, that this equi- librium temperature is well below the ambient air temperature $T_{a}$ of $27^{\circ} \mathrm{C}$. Very little evaporation occurs, however, until at least $10 \mathrm{~s}$ after formation; and the droplet does not reach its equilibrium radius until at least 100 s after its formation. The final point to notice in Fig. 1 is that this equilibrium radius is not zero. Because the droplet is saline, it retains some liquid as long as the relative humidity is $75 \%$ or higher (Twomey 1953; Pruppacher and Klett 1978, Table 4.3). Below that humidity, it will crystallize into a salt particle.

Andreas (1995) showed plots like Fig. 1 for other conditions, and Andreas (1990, 1992) explained that the three-order-of-magnitude difference in timescales for temperature and radius evolution that is apparent for the $100-\mu \mathrm{m}$ droplet in Fig. 1 also manifests in all spray droplets with radii between 1 and $500 \mu \mathrm{m}$. In other words, the sensible and latent heat exchanges from spray droplets are decoupled. The ambient humidity has negligible effect on the temperature timescale; and the sea surface temperature $T_{s}$ (also the initial droplet temperature) has no effect on the radius timescale because the droplet is at its equilibrium temperature $T_{\mathrm{eq}}$ during most of its evaporation.

These essential facts lead us to believe that spray droplets with relatively short atmospheric residence times - that is, re-entrant spray-can accomplish a net sea-to-air enthalpy transfer. Our scenario relies on the rapidity with which droplets reach thermal equilibrium coupled with the much slower rate at which they evaporate. In other words, droplets readily give up heat associated with the falling temperature trace in Fig. 1, but many fall back into the sea before extracting from the atmosphere the latent heat to evaporate as represented by the radius trace in Fig. 1. The recent microphysical modeling by Van Eijk et al. (2001) corroborates this scenario that most spray droplets reach their equilibrium temperature but fail to reach their equilibrium radius before falling back into the sea.

Most others who have considered this problem have discounted the sensible heat carried by the spray and have focused on the latent heat transfer because this is, potentially, a much larger flux (e.g., Fairall et al. 1990; Hasse 1992; Makin 1998). But as Hasse (1992) and Emanuel (1995) pointed out, to evaporate completely a spray droplet must extract as much sensible heat from the near-surface droplet evaporation layer (Andreas et al. 1995) as it gives up in latent heat. That is, if a spray droplet were to evaporate entirely, its evaporation would reflect no net sea-air enthalpy transfer, only a conversion between the latent and sensible heat contributions to enthalpy.

We demonstrate the role of the sensible heat carried by the spray droplets in accomplishing a net sea-air enthalpy exchange in Fig. 2. Consider a system consisting of a near-surface layer of the ocean initially at temperature $T_{s}$ underneath a near-surface air layer initially at temperature $T_{a}$. The near-surface ocean has initial mass $m_{0}$; the near-surface air initially contains no 


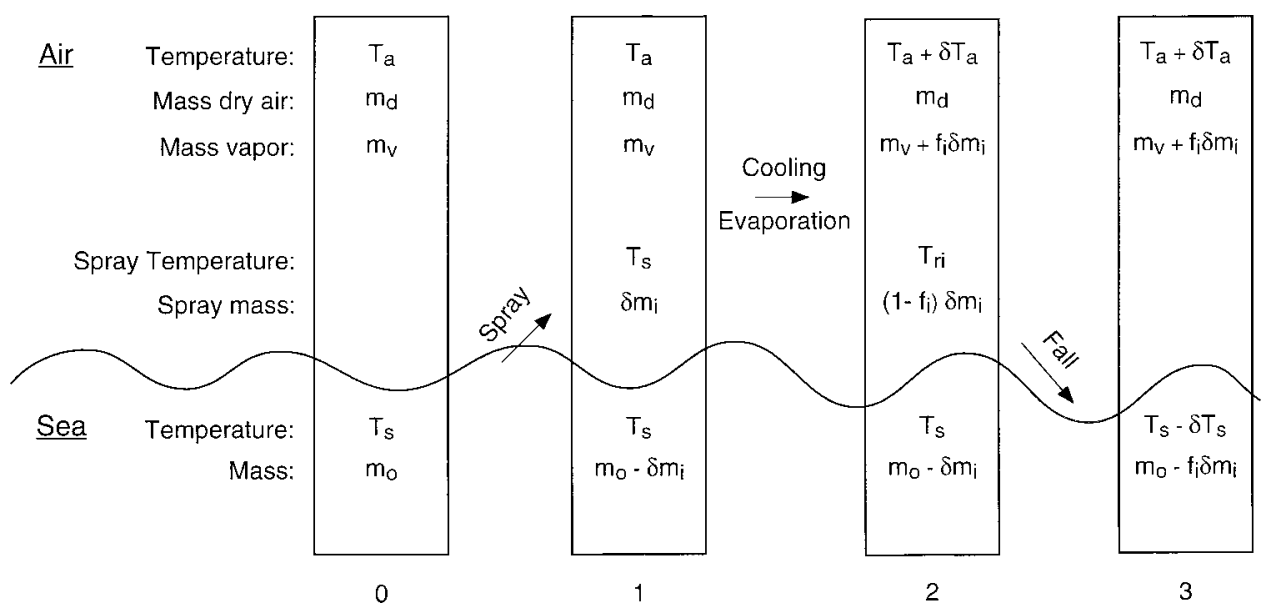

FIG. 2. A schematic diagram of the thermodynamic steps that occur in a control volume enclosing the near-surface ocean and the near-surface atmosphere when the ocean ejects spray, the spray cools and evaporates, and then falls back into the ocean.

spray and has dry air mass $m_{d}$ and water vapor mass $m_{v}$ (Fig. 2, panel 0). In panel 1, we let a small amount of ocean mass $\delta m_{i}$ be converted to spray droplets of radius $r_{i}$. This step adds mass with temperature $T_{s}$ to the air and diminishes the ocean mass by $\delta m_{i}$. Actually, spray droplets have a wide range of sizes, as we mentioned. Subscript " $i$ " here means that we consider only a small, arbitrary radius bin for this demonstration.

In panel 2 of Fig. 2, we let the spray droplets cool to $T_{r i}$ and let a fraction $f_{i}$ of the original spray mass evaporate. We assume that $T_{r i}$ here is the droplet equilibrium temperature (see Fig. 1) because all droplets up to $500 \mu \mathrm{m}$ in radius reach their equilibrium temperature within $5 \mathrm{~s}$, which is generally shorter than their residence time when the winds are above $20 \mathrm{~m} \mathrm{~s}^{-1}$ (Andreas et al. 1995). The cooling and evaporation do not change the properties of the ocean, but they do change the air temperature by $\delta T_{a}$. The evaporation also adds vapor in the amount $f_{i} \delta m_{i}$ to the air. Since the droplets have given up sensible heat to the air during their cooling but have extracted some sensible heat to evaporate, we cannot be sure of the sign of $\delta T_{a}$. Does the spray warm or cool the air? Most believe that spray cools the air; but the advantage of this thought experiment is that we do not have to know.

Finally, in panel 3, all the spray has fallen back into the sea. The air remains at temperature $T_{a}+\delta T_{a}$ and with vapor mass $m_{v}+f_{i} \delta m_{i}$; but this step clearly cools the ocean by $\delta T_{s}$ because the re-entrant spray is at $T_{r i}$, which is less than both $T_{a}$ and $T_{s}$ (see Fig. 1). Because enthalpy must be conserved, by cooling the ocean, the spray has obviously accomplished a net enthalpy transfer from the sea to the air. In other words, by comparing the state of the air in panels 0 and 2 in Fig. 2, we cannot tell what net effect the spray has had. Such focus on the near-surface air has been the source of most of the confusion over spray's role in air-sea exchange. But when we compare the state of the ocean in panels 0 and
3 , spray's role is clear. The spray has cooled the ocean and, thus, must have accomplished a net enthalpy transfer from the sea to the air. (Note, however, that this cooling of the ocean does not constitute a large sink term in the ocean's thermal budget, especially when compared to other effects such as vertical mixing through the base of the oceanic mixed layer.)

One necessary clarification for panel 3 is that the smallest spray droplets do not fall back into the sea. These droplets have very long residence times and thus would contribute little, if any, to the sea-air enthalpy exchange. But Fig. 2 accurately depicts the fate of droplets larger, nominally, than $10 \mu \mathrm{m}$, which carry most of the spray sensible and latent heat (e.g., Andreas 1992; Andreas et al. 1995; Van Eijk et al. 2001).

We can formalize the scenario depicted in Fig. 2 by evaluating the total system enthalpy. In panel 0 , the system enthalpy is

$$
K_{0}=\left(c_{p d} m_{d}+c_{w} m_{v}\right) T_{a}+L_{v} m_{v}+c_{w} m_{0} T_{s},
$$

where $c_{p d}$ and $c_{w}$ are the heat capacities of dry air and liquid water, and $L_{v}$ is the latent heat of vaporization. The first two terms on the right in (1) represent the total enthalpy of the air, while the third term is the enthalpy of the ocean water.

In panel 2, the system enthalpy is

$$
\begin{aligned}
K_{2}= & {\left[c_{p d} m_{d}+c_{w}\left(m_{v}+f_{i} \delta m_{i}\right)\right]\left(T_{a}+\delta T_{a}\right) } \\
& +\left(L_{v}+\delta T_{v}\right)\left(m_{v}+f_{i} \delta m_{i}\right)+c_{w} \delta m_{i}\left(1-f_{i}\right) T_{r i} \\
& +c_{w}\left(m_{o}-\delta m_{i}\right) T_{s},
\end{aligned}
$$

where $\delta L_{v}$ is the change in the latent heat of vaporization resulting from the change in air temperature.

Since the total enthalpy of the system must be conserved, $K_{0}$ and $K_{2}$ must be equal. Equating these gives

$$
\begin{aligned}
& {\left[c_{p d} m_{d}+c_{p v}\left(m_{v}+f_{i} \delta m_{i}\right)\right] \delta T_{a}+L_{v} f_{i} \delta m_{i}} \\
& \quad=c_{w} \delta m_{i}\left[\left(T_{s}-T_{a}\right)+\left(1-f_{i}\right)\left(T_{a}-T_{r i}\right)\right],
\end{aligned}
$$


where $c_{p v}$ is the heat capacity at constant pressure of water vapor, and we have used $\delta L_{v}=\left(c_{p v}-c_{w}\right) \delta T_{a}$. Our convention throughout this development has been that $\delta T_{a}, \delta m_{i}$, and $\delta L_{v}$ all have their probable signs: $\delta T_{a}<0, \delta m_{i}>0$, and $\delta L_{v}<0$.

The left side of (3) is the change in enthalpy of the air between panels 0 and 2 in Fig. 2; the right side shows the processes that accomplish this change. The enthalpy of the air increases because of the sensible heat that all droplets give up in falling from their initial temperature $T_{s}$ down to the air temperature $T_{a}$. The enthalpy of the air also increases because of the sensible heat lost when the droplet's temperature falls from temperature $T_{a}$ to $T_{r i}$.

In the final panel in Fig. 2, the remaining spray falls back into the ocean. This step changes neither the temperature nor the specific humidity of the air and thus does not affect the air's specific enthalpy. The ocean does cool in this process, however, reflecting the net enthalpy transfer to the atmosphere.

We can draw several important conclusions from (3). If the entire droplet evaporates $\left(f_{i}=1\right)$, the net enthalpy gain comes only from bringing the spray from ocean temperature to ambient air temperature. Freshwater droplets may evaporate entirely, but larger seawater droplets rarely do. Therefore, even if the ocean and atmosphere have the same temperature, there can still be a net enthalpy transfer since $f_{i}<1$ is the usual case for seawater droplets. In general, if there is no evaporation, the droplet's temperature will be somewhere between $T_{s}$ and $T_{a}$, and there will be a small net enthalpy transfer.

But the major effect comes when evaporation reduces the droplet temperature to $T_{r i}$. Consider a droplet with initial mass $m_{i}$ and temperature $T_{s}$. The droplet cools from $T_{s}$ to the air temperature primarily by losing sensible heat. To cool from $T_{a}$ to $T_{r i}$, however, it must evaporate some of its water. We ask how much droplet mass must evaporate for the droplet to cool from $T_{a}$ to $T_{r i}$. The heat balance of this cooling is simply

$$
m_{i} c_{w}\left(T_{a}-T_{\text {eq }}\right)=L_{v}\left(m_{i}-m\right),
$$

where $m$ is the droplet's mass when its temperature reaches $T_{\text {eq } i}$. Clearly,

$$
\frac{m}{m_{i}}=1-\frac{c_{w}}{L_{v}}\left(T_{a}-T_{\text {eq } i}\right) .
$$

Therefore, if $T_{a}-T_{\text {eq } i} \approx 3^{\circ} \mathrm{C}$, for example (cf. Fig. 1 ), $m / m_{i} \approx 0.995$. That is, less than $1 \%$ of the droplet's initial mass must evaporate for it to cool well below $T_{a}$. Notice how rapid this process is. According to Fig. 1, a droplet with an initial radius of $100 \mu \mathrm{m}$ reaches $T_{\text {eq } i}$ in less than a second. Larger droplets take somewhat longer; smaller droplets evolve even more quickly (see also Andreas 1990; Andreas and DeCosmo 1999).

The exact temperature that the droplet attains depends on its initial size, salt content, ambient temperature and humidity, and length of time in the air; but the equili- bration timescale is typically very short compared to the average time a droplet spends airborne (e.g., Andreas 1992). Thus, to a first approximation, we may consider $T_{r i}$ to be the wet-bulb temperature of a saline, spherical droplet of radius $r_{i}$. The largest heat transfer will occur when the fraction of droplet that evaporates, $f_{i}$, is just large enough to bring the droplet temperature down close to $T_{r i}$. Again, this fraction is of order $1 \%$.

\section{The net thermodynamic contribution of spray}

A tropical cyclone responds to the net enthalpy transfer across the air-sea interface rather than to the individual fluxes of sensible and latent heat. Our hypothesis here is that re-entrant sea spray contributes some of that enthalpy flux. In the context of Andreas's (1992) spray model, Andreas and DeCosmo (1999) write the total air-sea enthalpy flux as

$$
Q_{e, T}=Q_{e, \mathrm{int}}+Q_{e, \mathrm{sp}} .
$$

Here, $Q_{e, \text { int }}$ is the usual interfacial enthalpy flux parameterized with the transfer coefficient $C_{k}$, and $Q_{e, \mathrm{sp}}$ is the spray enthalpy flux.

In light of our discussion in the last section, we formulate $Q_{e, s p}$ as a sensible heat flux driven by the temperature difference between the sea surface and the spray droplets at their thermal equilibrium. Andreas (1992) and Andreas et al. (1995), however, show that spray droplets with an initial radius of about $100 \mu \mathrm{m}$ carry most of the spray sensible heat. Therefore, rather than considering droplets of all sizes (each with a unique $T_{\mathrm{eq}}$ ), to make the parameterization simple, we hypothesize that these $100-\mu \mathrm{m}$ droplets are the bellwethers of the spray enthalpy transfer and thus parameterize $Q_{e, s p}$ as

$$
Q_{e, \mathrm{sp}}=\rho_{w} c_{w}\left(T_{s}-T_{\mathrm{eq}, 100}\right) V\left(u_{*}\right) .
$$

Here, $\rho_{w}$ is the density of seawater, $V\left(u_{*}\right)$ is a function of the friction velocity $u_{*}$, and $T_{\text {eq, } 100}$ is the equilibrium temperature of droplets that are initially $100 \mu \mathrm{m}$ in radius. In actual practice, though, we approximate this equilibrium temperature with the salinity-modified wetbulb temperature. (For $u_{*}$ in $\mathrm{m} \mathrm{s}^{-1}$ and for the other variables on the right-hand side of (7) in mks units, $Q_{e, s p}$ is in $\mathrm{W} \mathrm{m}^{-2}$.)

Andreas and DeCosmo (1999) extracted spray sensible and latent heat fluxes from DeCosmo's (1991) HEXOS data using Andreas's (1992) spray generation function to predict spray production. Andreas and Emanuel (1999) compared the spray enthalpy flux $Q_{e, \mathrm{sp}}$ implied by this partitioning with the HEXOS measurements of the total enthalpy flux $Q_{e, T}$. Figure 3 likewise shows that spray enthalpy flux but parameterized here as (7) suggests.

The rate at which the wind does work on the sea surface to produce spray droplets has long been presumed to go approximately as the cube of the wind speed or the cube of $u_{*}$ (e.g., Wu 1979; Monahan et al. 1986; Monahan 1988; Andreas et al. 1995; Andreas 1998a). 


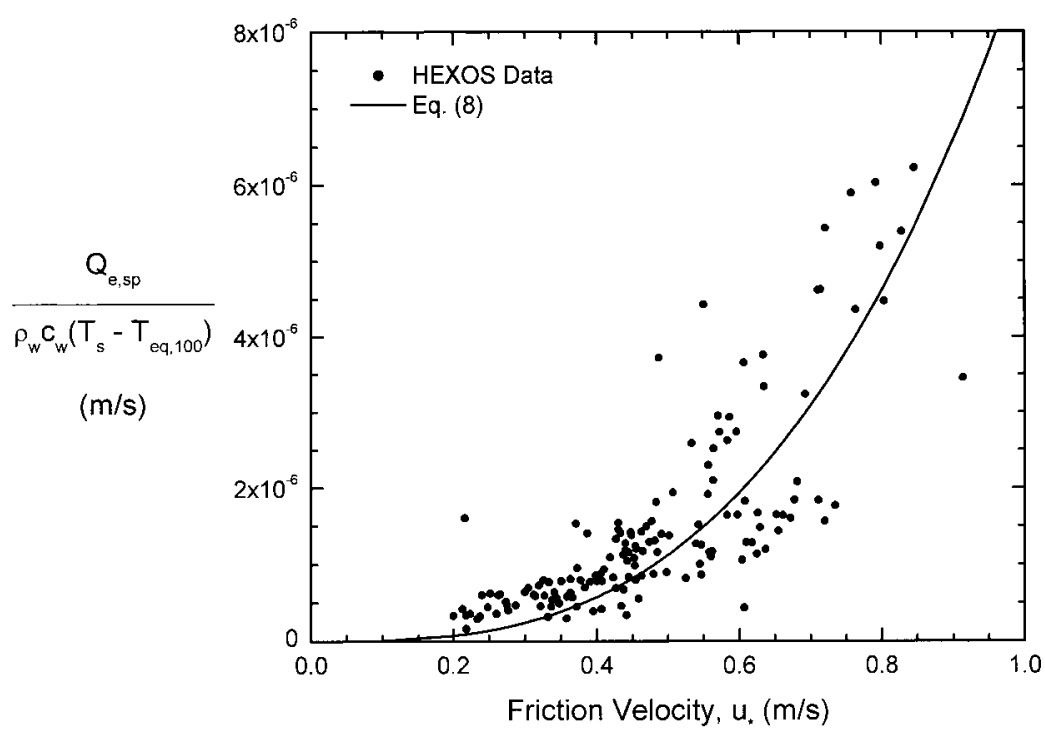

FIG. 3. The spray enthalpy flux that Andreas and DeCosmo (1999) inferred from the HEXOS data is plotted as suggested in (7) to deduce the wind function $V\left(u_{*}\right)$, which is given by (8).

We fitted the line in Fig. 3 to the HEXOS data by doing a least squares fit for an assumed wind dependence of $u_{*}^{3}$. The result is

$$
V\left(u_{*}\right)=9.0 \times 10^{-6} u_{*}^{3}
$$

$\left(V\right.$ in $\mathrm{m} \mathrm{s}^{-1}$ for $u_{*}$ in $\left.\mathrm{m} \mathrm{s}^{-1}\right)$.

As an example of the magnitude of the spray enthalpy flux, suppose the water temperature is $28^{\circ} \mathrm{C}$, the air temperature is $27^{\circ} \mathrm{C}$, and the relative humidity is $80 \%$ (cf. Fig. 1). The equilibrium temperature of a $100-\mu \mathrm{m}$ spray droplet with initial salinity 34 psu is then $24.36^{\circ} \mathrm{C}$ (e.g., Andreas 1995, 1996; Kepert 1996). For a 10-m wind speed of $20 \mathrm{~m} \mathrm{~s}^{-1}$, we use Large and Pond's (1981) results to estimate that the $10-\mathrm{m}$ drag coefficient is 1.79 $\times 10^{-3} ; u_{*}$ is consequently $0.84 \mathrm{~m} \mathrm{~s}^{-1}$. Equations (7) and (8) then give $Q_{e, s \mathrm{sp}}=79 \mathrm{~W} \mathrm{~m}^{-2}$. This is already a large number. To the extent that we can extrapolate (7) and (8) to cyclone-strength winds, the spray enthalpy flux could be very large indeed. We suggest that these spray effects may be the source of the anomalous surface heat fluxes that tropical cyclones require for their generation and maintenance (Emanuel 1995; Smith 1997).

\section{The spray momentum flux}

When spray is ejected into the airstream, air drag accelerates it. This exchange extracts momentum from the airflow. Likewise, when spray droplets crash back into the sea, they transfer their momentum to the ocean. For moderate winds, however, Wu (1973) found this spray-mediated momentum exchange to be negligible. Fairall et al. (1994) likewise found the effect negligible for winds up to $50 \mathrm{~m} \mathrm{~s}^{-1}$. Pielke and Lee (1991), on the other hand, suggest that the spray drag is significant. In their model, it reduces the near-surface wind speed by $15 \%$ at a wind speed of $40 \mathrm{~m} \mathrm{~s}^{-1}$ and, consequently, would increase the surface stress by a few percent. We revisit this issue of spray momentum here.

By integrating the equation for the horizontal force balance on a spray droplet, we have calculated how long a droplet of radius $r_{0}$ takes to accelerate from zero to within $e^{-1}$ of the local wind speed. We find that, in surface-level winds of $10 \mathrm{~m} \mathrm{~s}^{-1}$ and higher, droplets with radii up to $500 \mu \mathrm{m}$ reach this speed within $1 \mathrm{~s}$. In turn, using Andreas's (1992) estimate of a droplet's residence time, we further see that all droplets up to 500 $\mu \mathrm{m}$ in radius are airborne long enough to accelerate to the local wind speed $U$.

We can next use this information to estimate the rate at which the spray extracts momentum from the airflow. Formally, that spray momentum flux is simply

$$
\tau_{\mathrm{sp}}=\frac{4 \pi}{3} \rho_{w} u \int_{r_{\mathrm{lo}}}^{r_{\mathrm{hi}}} r_{0}^{3} \frac{d F}{d r_{0}} d r_{0},
$$

where $d F / d r_{0}$ is the so-called spray generation function, the number of droplets of initial radius $r_{0}$ produced per square meter of sea surface per second per micrometer increment in $r_{0}$. Consequently, in (9), $\left(4 \pi \rho_{w} r_{0}^{3} / 3\right) d F / d r_{0}$ is the mass flux of spray droplets with radius $r_{0}$. The limits of integration, $r_{\mathrm{lo}}$ and $r_{\mathrm{hi}}$, are nominally 1 and 500 $\mu \mathrm{m}$.

For $U$ in (9), we use the wind speed at one significant wave height above mean sea level. That is, (9) assumes that all droplets reach this level. This is a good assumption because the droplets that contribute most to (9) are the large ones torn right off the wave crests.

Equation (9) is also an upper bound on the surface stress that falling spray imparts to the ocean. It is only an upper bound, though, because some of the smaller 


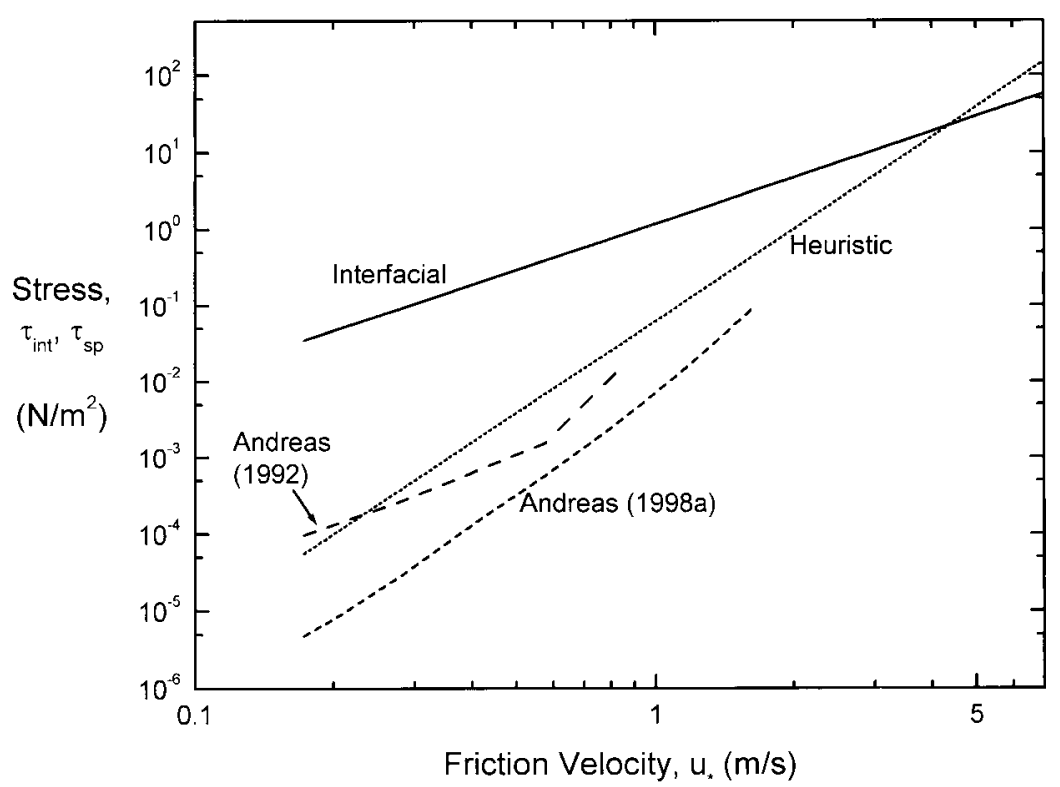

FIG. 4. Estimates of the interfacial and spray stresses. The domain of the spray stresses calculated using (9) and the Andreas (1992) and Andreas (1998a) spray generation functions reflects the wind speed limits over which these functions are defined. The heuristic line is Eq. (11).

spray droplets remain suspended indefinitely and thus do not fall back into the sea. Some of the droplets also leave water vapor behind in the atmosphere and therefore do not return to the sea with radius $r_{0}$. Nevertheless, the essential point is that this transfer of momentum from air to sea through the spray is immaterial to the dynamics of the cyclone; the cyclone responds only to the spray-mediated momentum exchange in the air, as estimated from (9).

Using (9), we have evaluated $\tau_{\mathrm{sp}}$ for the Andreas (1992) and Andreas (1998a) spray generation functions. Figure 4 shows the results of these calculations. For comparison, the figure also shows the interfacial surface stress, which is just

$$
\tau_{\text {int }}=\rho_{a} u_{*}^{2},
$$

where $\rho_{a}$ is the air density.

Figure 4 suggests that, for moderate winds, the spray stress implied by the Andreas (1992) and Andreas (1998a) spray generation functions is two to three orders of magnitude less than the interfacial stress, as Wu (1973) concluded. But the estimated spray stress increases roughly as the fourth power of $u_{*}$ because $d F /$ $d r_{0}$ is approximately cubic in $u_{*}$ (Andreas 1998a) and $U$ is linear in $u_{*}$. Meanwhile, $\tau_{\text {int }}$ is quadratic in $u_{*}$. We estimate that, when the winds reach hurricane strength, the interfacial and spray stresses become comparable.

The two estimates of spray stress in Fig. 4 set a lower bound on the actual spray stress because they model only droplets with initial radii between 1 and $500 \mu \mathrm{m}$. Spume droplets can be much larger than this. And though these very large droplets contribute negligibly to the enthalpy transfer, they should enhance the spray momentum transfer beyond what we predict with the Andreas (1992) and Andreas (1998a) spray generation functions.

Therefore, the line labeled "Heuristic" in Fig. 4 depicts our current parameterization for the spray stress,

$$
\tau_{\mathrm{sp}}=6.2 \times 10^{-2} u_{*}^{4}
$$

$\left(\tau_{\mathrm{sp}}\right.$ in $\mathrm{N} \mathrm{m}^{-2}$ for $u_{*}$ in $\mathrm{m} \mathrm{s}^{-1}$ ). As the figure shows, the interfacial stress and this spray stress are equal at about $u_{*}=4.2 \mathrm{~m} \mathrm{~s}^{-1}$; this $u_{*}$ corresponds to a surface-level wind speed of about $60 \mathrm{~m} \mathrm{~s}^{-1}$. For higher wind speeds, the spray stress actually exceeds the interfacial stress.

\section{Tests using a simple model}

As a preliminary test of the new spray formulations, we incorporate them in the simple, balanced axisymmetric model of Emanuel (1995). This model is phrased in potential radius coordinates and thus yields extremely high horizontal resolution in the eyewall region, where the vorticity is large. It also uses a representation of convection based on the subcloud layer entropy equilibrium postulate. The interfacial fluxes of enthalpy and momentum are calculated using standard bulk-aerodynamic formulas but with coefficients that depend linearly on wind speed (but are always equal to each other), as we explain in the appendix. The environmental conditions used in all of these simulations are those of the standard control run in Emanuel (1995); that is, the sea surface temperature is $27^{\circ} \mathrm{C}$, the mean outflow temper- 
TABLE 1. Summary of the four simulations depicted in Fig. 5.

\begin{tabular}{lcccc}
\hline \hline \multicolumn{1}{c}{ Simulation } & Interfacial stress & Interfacial enthalpy & Spray enthalpy & Spray stress \\
\hline Control & $C_{D}=C_{k}$ and both increase linearly with wind speed & & & \\
Wave drag only & Use Eqs. (A2), (A5), and (A6) & Use Eqs. (A8), (A9), and (A10) & & \\
Spray enthalpy and momentum & $C_{D}=C_{k}$ and both increase linearly with wind speed & Use Eqs. (7) and (8) & Use Eq. (11) \\
All effects & Use Eqs. (A2), (A5), and (A6) & Use Eqs. (A8), (A9), and (A10) & Use Eqs. (7) and (8) & Use Eq. (11) \\
\hline
\end{tabular}

ature is $-70^{\circ} \mathrm{C}$, and the mean ambient surface-level relative humidity is $80 \%$.

To model the spray enthalpy and momentum fluxes, we add (7), (8), and (11) to the model's standard bulkflux formulation and assume that $T_{\text {eq, } 100}$ is the wet-bulb temperature (modified for salinity) of the well-mixed boundary layer air at a height of $10 \mathrm{~m}$. We also incorporate in the model the effects of wave drag on the interfacial momentum and enthalpy fluxes as described in the appendix. In all other respects, the model is identical to that used in Emanuel (1995). Note that Emanuel's model requires only the net enthalpy flux from the sea surface; temperature and moisture are not carried as separate variables. Since moist enthalpy is the sole thermodynamic variable of the model, we need not keep track of the air-sea temperature difference and the moisture gradient separately.

We run four hurricane simulations: the standard (i.e., control) simulation reported in Emanuel (1995), which uses bulk exchange coefficients $C_{k}$ and $C_{D}$ that depend linearly on wind speed but are equal to each other at all wind speeds; a simulation using the Charnock re- lation (A6) and the associated scalar roughness length (A10) to represent wave drag but with no spray effects; a simulation using (7), (8), and (11) for the spray enthalpy and momentum fluxes but with no wave drag; and a simulation using wave drag, (A6) and (A10), together with (7), (8), and (11) for the spray enthalpy and momentum fluxes. Table 1 summarizes the details of these simulations; Fig. 5 shows our results.

It is clear from Fig. 5 that the wave drag effect, uncompensated by any enhanced surface enthalpy flux, leads to a substantial diminution of storm intensity. In fact, the model cannot generate very intense hurricanes even in very favorable environments. While it is unreasonable to suppose that the sea state is in equilibrium in a hurricane-as assumed in the Charnock formulaactual sea states might exert even more drag because waves are steeper and have lower average phase speeds before they reach equilibrium.

When we include the spray enthalpy and momentum effects in the third simulation in Fig. 5, storm intensity increases significantly because of the enhanced enthalpy exchange associated with the re-entrant sea spray. We

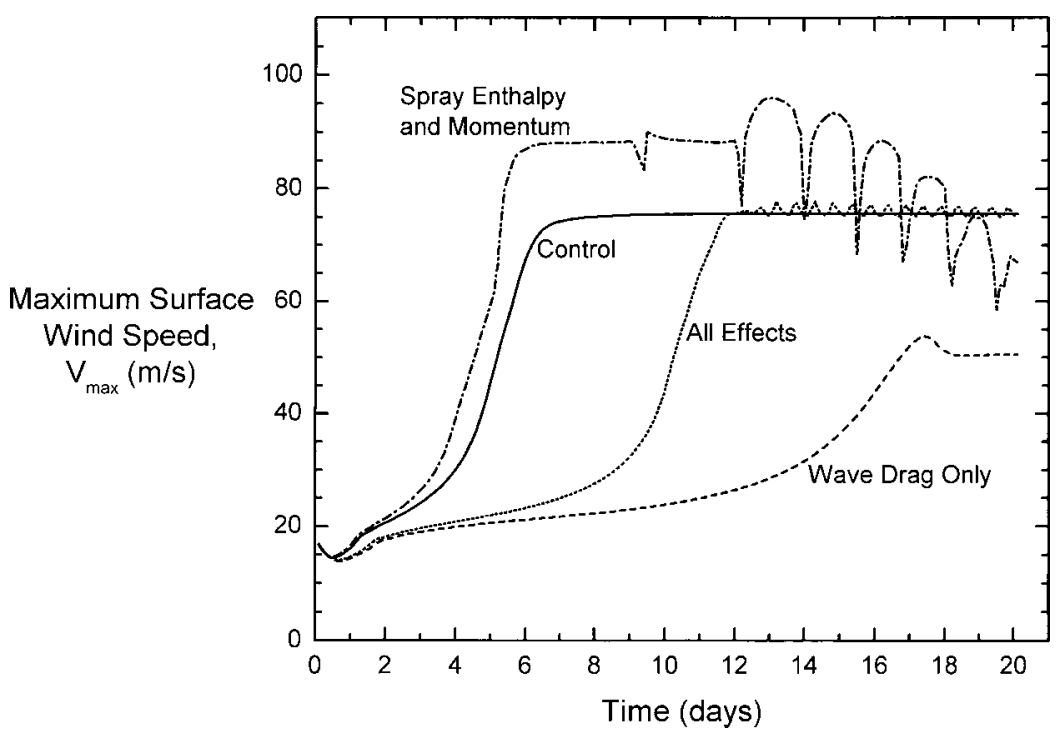

FIG. 5. Evolution with time of the maximum surface wind speed, $V_{\max }$, in four integrations of Emanuel's (1995) tropical cyclone model. The "Control" run uses identical exchange coefficients for enthalpy and momentum. The "Wave Drag Only" run simulates wave effects on the drag coefficient using the Charnock relation, (A6), and a comparable parameterization for the scalar roughness, (A10). The "Spray Enthalpy and Momentum" run models spray enthalpy and momentum fluxes using (7), (8), and (11) but includes no wave drag. The run with "All Effects" includes both the spray and wave parameterizations. See Table 1 . 
are not really surprised that this spray simulation produces such an intense storm. As Table 1 shows, this simulation simply adds an enthalpy flux mediated by the spray to the control run, which already has enough enthalpy transfer to produce a realistic storm. The spray momentum flux does moderate the effects of the enhanced enthalpy flux in this simulation, though.

The oscillatory pattern in the spray simulation results from eyewall replacement cycles, during which the hurricane eyewall collapses and reforms (e.g., Bister and Emanuel 1998). Eyewall replacement cycles tend to occur in this model when either the ambient middle troposphere is exceptionally moist or the surface enthalpy flux is exceptionally strong (Emanuel 1995). We could eliminate the eyewall cycles here, without affecting the average intensity of the modeled storm, by making the middle troposphere drier.

While our formulations of the spray fluxes, (7), (8), and (11), must be regarded as highly provisional, it is clear that, based on our current understanding, sea spray can have a significant effect on storm intensity. When we include both wave drag and spray enthalpy and momentum exchange in the fourth simulation in Fig. 5 (i.e., "All Effects"), the model reaches an intensity similar to that of the control run. That is, in this case, the wave drag and sea spray effects nearly cancel. This perhaps explains why models with very simple formulations of air-sea exchange produce simulated storms of reasonable intensity (Emanuel 1999).

\section{Summary}

It is clear from both theory and numerical simulations that the observed intensity of tropical cyclones cannot be explained by air-sea interaction physics that accounts only for increased wave drag without an attendant process that enhances the sea-air enthalpy flux. We here propose that the "missing" process is re-entrant sea spray. Spray droplets injected into the air thermally equilibrate after only about $1 \%$ of their mass has evaporated. When they fall back into the sea before losing much more of their mass, they give up to the atmosphere sensible heat before they have had time to extract a higher price in latent heat. The HEXOS measurements support a cubic dependence of the spray enthalpy flux on friction velocity. Extrapolating these results to hurricane-force winds in a numerical model shows that the spray-induced enthalpy flux can be very significantmore than enough to offset the increased drag caused by the spray and potentially enough to offset the increased wave drag as well.

We are evidently the first to incorporate this spray drag into a tropical cyclone model. Though previous estimates have suggested that spray droplets have negligible influence on air-sea momentum exchange for moderate winds, our analysis of spray generation rates suggests that the spray drag and the interfacial drag become comparable in hurricane-strength winds. Quite simply, the interfacial drag increases quadratically with the friction velocity, while the spray drag appears to increase as the fourth power. We estimate that the two momentum transfer mechanisms are thus equal for a surface-level wind speed of about $60 \mathrm{~m} \mathrm{~s}^{-1}$.

Acknowledgments. We thank three anonymous referees for helpful reviews. Ed Andreas received support from the Office of Naval Research through Contracts N0001498 MP30029, N00014992MP30043, N0001400MP20031, and N0001401MP20042, and from the National Science Foundation through Grant ATM-00-01037. Kerry Emanuel received support through NSF Grant ATM-00-01039.

\section{APPENDIX}

\section{Gradient-Wind Transfer Laws}

The wind speed variable in Emanuel's (1995) tropical cyclone model is the gradient wind. Consequently, the standard version of that model parameterizes the interfacial fluxes of momentum and enthalpy in terms of transfer coefficients $C_{D}$ and $C_{k}$, respectively, that apply to the gradient wind. In our control run here, $C_{D}$ and $C_{k}$ increase linearly with the wind speed but are always equal.

For other runs mentioned in the text that involve spray and wave effects, however, we formulate the spray and interfacial exchanges in terms of the friction velocity $u_{*}$ since this is, dynamically, a more fundamental variable than the usual $10-\mathrm{m}$ wind speed. We thus need transfer laws that relate the gradient wind and the enthalpy at the gradient-wind level to the interfacial momentum and enthalpy fluxes. For example, in the "wave drag" and "all effects" runs, we model the interfacial momentum flux as

$$
\tau_{\text {int }}=\rho_{a} u_{*}^{2},
$$

where $\rho_{a}$ is the air density. We therefore need a gradientwind transfer coefficient, $C_{D g}$, that relates $u_{*}$ to the magnitude of the gradient wind, $G$ :

$$
u_{*}=C_{D g} G
$$

Likewise, in these same runs, we model the interfacial enthalpy flux as

$$
Q_{e, \mathrm{int}}=-\rho_{a} u_{*} k_{*},
$$

where $k_{*}$ is the enthalpy flux scale (comparable to the temperature and moisture flux scales of Monin-Obukhov similarity theory). We thus also need an enthalpy flux transfer coefficient, $C_{k g}$, that relates $k_{*}$ to the difference between the enthalpy at the sea surface, $K_{s}$, and the enthalpy at the level of the gradient wind, $K_{a}$ :

$$
-k_{*}=C_{k g}\left(K_{s}-K_{a}\right) .
$$

We derive these transfer coefficients using Rossby similarity and two assumptions: i) in the boundary layer of a tropical cyclone, the gradient wind takes the place 
of the geostrophic wind in forcing surface exchange; ii) because of the high winds, the boundary layer stratification is near neutral.

Under these assumptions, we can simply write down the drag coefficient that Andreas (1998b) obtained,

$$
C_{D g}=\frac{\kappa}{\left\{\left[\ln \left(h_{g} / z_{0}\right)-A(0)\right]^{2}+B(0)^{2}\right\}^{1 / 2}} .
$$

Here, $\kappa(=0.40)$ is the von Kármán constant; $h_{g}$ is the reference height for the gradient wind; and $A(0)$ and $B(0)$ are the neutral-stability values of the Rossby similarity functions for longitudinal and transverse wind components, as defined by Yamada (1976), for example. Yamada gives $A(0)=1.855$ and $B(0)=3.020$, but here we use the values from Zilitinkevich's (1989a) more recent review, $A(0)=1.7$ and $B(0)=4.5$.

To represent the effect of wave drag, we parameterize the roughness length $z_{0}$ in (A5) with Charnock's relation,

$$
z_{0}=\alpha \frac{u_{*}^{2}}{g} .
$$

Here, $g$ is the acceleration of gravity; and $\alpha$ is the Charnock constant, which we take as 0.0185 (Wu 1982; Johnson et al. 1998).

The value for $C_{k g}$ in (A4) is likewise derived from Rossby similarity. From Andreas (1998b), we see that

$$
-k_{*}=\frac{\kappa\left(K_{s}-K_{a}\right)}{\ln \left(h_{k} / z_{k}\right)-C(0)} .
$$

Here, $h_{k}$ is the height at which we evaluate $K_{a}, z_{k}$ is the roughness length for enthalpy, and $C(0)$ is the neutralstability value of the Rossby similarity function for temperature (e.g., Yamada 1976). We take $C(0)=3.7$ (Yamada 1976; Zilitinkevich 1989b). Hence, from (A4) and (A7), the enthalpy transfer coefficient is

$$
C_{k g}=\frac{\kappa}{\ln \left(h_{k} / z_{k}\right)-C(0)}
$$

then

$$
Q_{e, \text { int }}=\rho_{a} C_{D g} C_{k g} G\left(K_{s}-K_{a}\right) .
$$

We identify $z_{k}$ in (A8) with the roughness length for temperature $\left(z_{T}\right)$ and use the COARE algorithm (Fairall et al. 1996) to estimate $z_{T}$. The scalar roughness $z_{T}$ is a decreasing function of the roughness Reynolds number $R_{*}=u_{*} z_{0} / v$, where $v$ is the kinematic viscosity of air. We compute $z_{0}$ and $u_{*}$ for $R_{*}$ from (A6) and (A2), respectively, but find that $z_{k}\left(=z_{T}\right)$ gets quite small rapidly. When $u_{*}$ is greater than $0.88 \mathrm{~m} \mathrm{~s}^{-1}$, the Coupled Ocean-Atmosphere Response Experiment (COARE) algorithm predicts that $z_{k}$ is smaller than the mean free path of an air molecule, about $7 \times 10^{-8} \mathrm{~m}$. It seems unphysical to us for $z_{k}$ to be any smaller than this. Hence, we simplify the predictions of the COARE algorithm for small $u_{*}$ and limit $z_{k}$ to the length of the mean free path for large $u_{*}$. Our $z_{k}$ algorithm is thus

$$
\begin{array}{ll}
z_{k}=2.27 \times 10^{-4} & \exp \left(-9.2 u_{*}\right) \\
& \text { for } 0 \leq u_{*} \leq 0.8788 \mathrm{~m} \mathrm{~s}^{-1}, \\
z_{k}=7.0 \times 10^{-8} & \text { for } 0.8788 \mathrm{~m} \mathrm{~s}^{-1}<u_{*} .
\end{array}
$$

In these, $z_{k}$ is in meters when $u_{*}$ is in meters per second. Note that because the $z_{0}$ value that went into these estimates acknowledges wave effect, $z_{k}$ does also.

\section{REFERENCES}

Andreas, E. L, 1990: Time constants for the evolution of sea spray droplets. Tellus, 42B, 481-497.

- 1992: Sea spray and the turbulent air-sea heat fluxes. J. Geophys. Res., 97, 11 429-11 441.

, 1995: The temperature of evaporating sea spray droplets. $J$. Atmos. Sci., 52, 852-862.

1996: Reply. J. Atmos. Sci., 53, 1642-1645.

1998a: A new sea spray generation function for wind speeds up to $32 \mathrm{~m} \mathrm{~s}^{-1}$. J. Phys. Oceanogr., 28, 2175-2184.

_ 1998 b: The atmospheric boundary layer over polar marine surfaces. Physics of Ice-Covered Seas, M. Leppäranta, Ed., Vol. 2, Helsinki University Press, 715-773.

_ , and J. DeCosmo, 1999: Sea spray production and influence on air-sea heat and moisture fluxes over the open ocean. Air-Sea Exchange: Physics, Chemistry and Dynamics, G. L. Geernaert, Ed., Kluwer, 327-362.

_- and K. A. Emanuel, 1999: Effects of sea spray on tropical cyclone intensity. Preprints, $23 d$ Conf. on Hurricanes and Tropical Meteorology, Dallas, TX, Amer. Meteor. Soc., 22-25.

- , and -2000 : How sea spray can affect the intensity of tropical cyclones. Preprints, 24th Conf. on Hurricanes and Tropical Meteorology, Fort Lauderdale, FL, Amer. Meteor. Soc., J17$\mathrm{J} 18$.

- J. B. Edson, E. C. Monahan, M. P. Rouault, and S. D. Smith, 1995: The spray contribution to net evaporation from the sea: A review of recent progress. Bound.-Layer Meteor., 72, 3-52.

Bao, J.-W., J. M. Wilczak, J.-K. Choi, and L. H. Kantha, 2000: Numerical simulations of air-sea interaction under high wind conditions using a coupled model: A study of hurricane development. Mon. Wea. Rev., 128, 2190-2210.

Bister, M., and K. A. Emanuel, 1998: Dissipative heating and hurricane intensity. Meteor. Atmos. Phys., 65, 233-240.

DeCosmo, J., 1991: Air-sea exchange of momentum, heat and water vapor over whitecap sea states. Ph.D. dissertation, University of Washington, Seattle, WA, 212 pp. [Available from UMI Dissertation Services, P. O. Box 1346, Ann Arbor, MI 48106-1346.]

_, K. B. Katsaros, S. D. Smith, R. J. Anderson, W. A. Oost, K. Bumke, and H. Chadwick, 1996: Air-sea exchange of water vapor and sensible heat: The Humidity Exchange over the Sea (HEXOS) results. J. Geophys. Res., 101, 12 001-12 016.

Edson, J. B., S. Anquentin, P. G. Mestayer, and J. F. Sini, 1996: Spray droplet modeling: 2. An interactive Eulerian-Lagrangian model of evaporating spray droplets. J. Geophys. Res., 101, 1279-1293.

Emanuel, K. A., 1986: An air-sea interaction theory for tropical cyclones. Part I: Steady-state maintenance. J. Atmos. Sci., 43, 585-604.

_ 1995 : Sensitivity of tropical cyclones to surface exchange coefficients and a revised steady-state model incorporating eye dynamics. J. Atmos. Sci., 52, 3969-3976.

— 1999: Thermodynamic control of hurricane intensity. Nature, 401, 665-669.

Fairall, C. W., J. B. Edson, and M. A. Miller, 1990: Heat fluxes, whitecaps, and sea spray. Surface Waves and Fluxes, G. L. Geernaert and W. J. Plant, Eds., Vol. 1, Current Theory, Kluwer, 173208.

, J. D. Kepert, and G. J. Holland, 1994: The effect of sea spray 
on surface energy transports over the ocean. Global Atmos. Ocean Syst., 2, 121-142.

- E. F. Bradley, D. P. Rogers, J. B. Edson, and G. S. Young, 1996: Bulk parameterization of air-sea fluxes for Tropical Ocean-Global Atmosphere Coupled Ocean-Atmosphere Response Experiment. J. Geophys. Res., 101, 3747-3764.

Geernaert, G. L., S. E. Larsen, and F. Hansen, 1987: Measurements of the wind stress, heat flux and turbulence intensity during strom conditions over the North Sea. J. Geophys. Res., 92, 13 127-13 139

Hasse, L., 1992: On the contribution of spray droplets to evaporation. Bound.-Layer Meteor., 61, 309-313.

Johnson, H. K., J. Højstrup, H. J. Vested, and S. E. Larsen, 1998: On the dependence of sea surface roughness on wind waves. $J$. Phys. Oceanogr., 28, 1702-1716.

Kepert, J. D., 1996: Comments on "The temperature of evaporating sea spray droplets." J. Atmos. Sci., 53, 1634-1641.

- C. W. Fairall, and J.-W. Bao, 1999: Modelling the interaction between the atmospheric boundary layer and evaporating sea spray droplets. Air-Sea Exchange: Physics, Chemistry and Dynamics, G. L. Geernaert, Ed., Kluwer, 363-409.

Korolev, V. S., S. A. Petrichenko, and V. D. Pudov, 1990: Heat and moisture exchange between the ocean and atmosphere in tropical storms Tess and Skip. Sov. Meteor. Hydrol., 3, 92-94.

Large, W. G., and S. Pond, 1981: Open ocean momentum flux measurements in moderate to strong winds. J. Phys. Oceanogr., 11, 324-336.

Lighthill, J., G. Holland, W. Gray, C. Landsea, G. Craig, J. Evans, Y. Kurihara, and C. Guard, 1994: Global climate change and tropical cyclones. Bull. Amer. Meteor. Soc., 75, 2147-2157.

Makin, V. K., 1998: Air-sea exchange of heat in the presence of wind waves and spray. J. Geophys. Res., 103, 1137-1152.

Mestayer, P. G., and C. Lefauconnier, 1988: Spray droplet generation, transport, and evaporation in a wind wave tunnel during the Humidity Exchange over the Sea Experiments in the Simulation Tunnel. J. Geophys. Res., 93, 572-586.

Monahan, E. C., 1988: Whitecap coverage as a remotely monitorable indication of the rate of bubble injection into the oceanic mixed layer. Sea Surface Sound, B. R. Kerman, Ed., Kluwer, 85-96.

- D. E. Spiel, and K. L. Davidson, 1986: A model of marine aerosol generation via whitecaps and wave disruption. Oceanic Whitecaps and Their Role in Air-Sea Exchange, E. C. Monahan and G. Mac Niocaill, Eds., D. Reidel, 167-174.

Ooyama, K., 1969: Numerical simulation of the life cycle of tropical cyclones. J. Atmos. Sci., 26, 3-40.

Pielke, R. A., and T. J. Lee, 1991: Influence of sea spray and rainfall on the surface wind profile during conditions of strong wind. Bound.-Layer Meteor., 55, 305-308.

Pruppacher, H. R., and J. D. Klett, 1978: Microphysics of Clouds and Precipitation. D. Reidel, 714 pp.

Riehl, H., 1954: Tropical Meteorology. McGraw-Hill, 392 pp.

Rosenthal, S. L., 1971: The response of a tropical cyclone model to variations in boundary layer parameters, initial conditions, lateral boundary conditions and domain size. Mon. Wea. Rev., 99, 767-777.

Rouault, M. P., P. G. Mestayer, and R. Schiestel, 1991: A model of evaporating spray droplet dispersion. J. Geophys. Res., 96, 71817200 .

Smith, R. K., 1997: On the theory of CISK. Quart. J. Roy. Meteor. Soc., 123, 407-418

Twomey, S., 1953: The identification of individual hygroscopic particles in the atmosphere by a phase-transition method. J. Appl. Phys., 24, 1099-1102.

Uang, C.-L., 1999: Impacts of sea spray and oceanic response on the development of tropical cyclones. Preprints, 23d Conf. on Hurricanes and Tropical Meteorology, Dallas, TX, Amer. Meteor. Soc., 30-31.

Van Eijk, A. M. J., B. S. Tranchant, and P. G. Mestayer, 2001: SeaCluse: Numerical simulation of evaporating sea spray droplets. J. Geophys. Res., 106, 2573-2588.

Wang, Y., J. D. Kepert, and G. J. Holland, 1999: The impact of sea spray evaporation on tropical cyclone intensification. Preprints, 23d Conf. on Hurricanes and Tropical Meteorology, Dallas, TX, Amer. Meteor. Soc., 26-29.

$\ldots$ _ _ _ and _ _ 2000: On the effect of sea spray evaporation on tropical cyclone boundary-layer structure and intensity. Preprints, 24th Conf. on Hurricanes and Tropical Meteorology, Fort Lauderdale, FL, Amer. Meteor. Soc., J19-J20.

Wu, J., 1973: Spray in the atmospheric surface layer: Laboratory study. J. Geophys. Res., 78, 511-519.

, 1979: Oceanic whitecaps and sea state. J. Phys. Oceanogr., 9, 1064-1068.

1982: Wind-stress coefficients over sea surface from breeze to hurricane. J. Geophys. Res., 87, 9704-9706.

Yamada, T., 1976: On the similarity functions A, B and C of the planetary boundary layer. J. Atmos. Sci., 33, 781-793.

Zilitinkevich, S. S., 1989a: Velocity profiles, the resistance law and the dissipation rate of mean flow kinetic energy in a neutrally and stably stratified planetary boundary layer. Bound.-Layer Meteor., 46, 367-387.

_ $1989 \mathrm{~b}$ : The temperature profile and heat transfer law in a neutrally and stably stratified planetary boundary layer. Bound.Layer Meteor., 49, 1-5. 